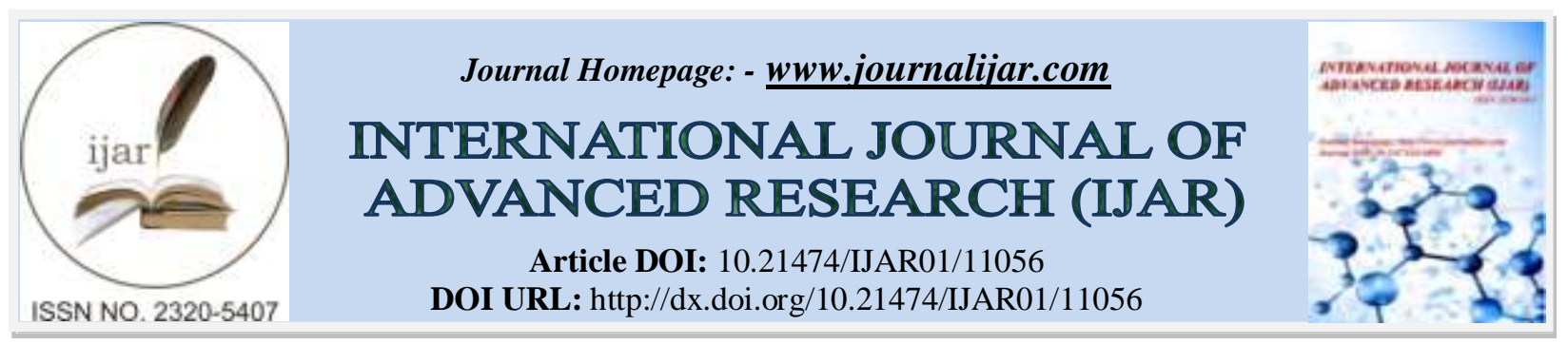

RESEARCH ARTICLE

\title{
A STUDY OF ENDOTHELIAL DYSFUNCTION IN PREDIABETICS AS EVIDENCED BY FLOW MEDIATED VASODILATION IN COLOR DOPPLER OF BRACHIAL ARTERY
}

Dr. T. Renuka and Dr. P. Rajendra

\section{Manuscript Info}

Manuscript History

Received: 25 March 2020

Final Accepted: 30 April 2020

Published: May 2020

\begin{abstract}
Endothelial dysfunction is an early event and it precedes atherosclerosis. Does endothelial functional assessment can provide valuable insight into "PRE-INTRUSIVE" phase of atherosclerosis . Flow mediated dilation is known to depend on ability of endothelium to release nitric oxide in response to shear stress and can be used to estimate reliably endothelial function."PRE-DIABETIC PATIENTS" have endothelial dysfunction due to HYPER GLYCAEMIA and INSULIN RESISTANCE. This study was under taken in pre diabetics to know the occurrence of endothelial dysfunction. Endothelial dysfunction was assessed Non Invasively by Doppler Ultrasound of brachial artery of pre diabetic patients who had impaired "Fasting Plasma Glucose". Flow mediated dilatation was calculated as percentage increase in brachial artery diameter in response to increase in brachial artery flow. The importance of unravelling mechanistic link between early insulin resistance and accelerated atherosclerosis is underscored by recent finding even in adolescents mild insulin resistance is associated with increased cardio vascular risk. Decreased nitric oxide bio- availability in endothelium is of particular relevance in pre-diabetics. This study was done with that hypothesis that insulin resistance is a substrate for accelerated atherosclerosis depicted by endothelial dysfunction. The findings in our study are that mild insulin resistance is associated with accelerated endothelial dysfunction.
\end{abstract}

Copy Right, IJAR, 2020,. All rights reserved.

\section{Introduction:-}

Criteria of pre diabetes:

1. Impaired fasting glucose ......... 100-125mg /dl

2. IFG WHO Guide lines........... 110-125mg/dl

3. Impaired Glucose .................. 140-199mg/dl tolerance test after $2 \mathrm{Hrs}$ post

4. HBA1C $5.7-6.4 \%$

Pre diabetes and cardiovascular disease:

1. IFG(100-125 mg/dl) CVD Risk 1.18

Corresponding Author:- Dr. P. Rajendra 
2. IFG(110-125mg/dl)

3. Impaired Glucose Tolerance
CVD Risk 1.20

CVD Risk 1.20

\section{Endothelial Cell dysfunction:}

It is described as imbalance between:

1. Relaxing factors and constructing factors

2. Anti-Coagulant mediators and Pro-coagulant mediators

3. Growth inhibiting factors and growth promoting factors

\section{Endothelial Cell dysfunction in Pre diabetics:}

Modulation of vascular tone, regulation of vessel inflammation and inhibition of thrombosis is impaired in pre diabetes , creating a permissive environment for atherogenesis

\section{Vasomotor function:}

Increased production of reactive oxygen species, decreased (NO) nitric oxide bioavailability of endothelium - derived $\mathrm{NO}$

\section{Inflammation:}

Insulin resistance increases inflammation, the " $\mathrm{T}$ " cells migrate to vascular intima. They secrete cytokines and chemokines recruiting monocytes and smooth muscle cells. Leading to fatty streaks.

\section{Thrombosis:}

Pre diabetic endothelial cells augment production of tissue factor, leading to thrombus formation.

\section{Biomarkers of endothelial cell Dysfunction:}

1. Elevated levels of plasma cellular adhesion molecules (E-selectin, I CAM 1 and V CAM 1), it raises relative risk of CVD in pre diabetes and diabetes by 1.5 - 7.5 fold.

2. Increased levels of inflammatory cytokines especially TNF , IL-6, CRP.

"Assessment of flow mediated dilation in brachial artery by color Doppler Ultrasonography"

The brachial artery flow mediated Doppler was performed in all subjects using $7.5 \mathrm{MHz}$ transducer. Test was done after overnight fasting. The B.P cuff was tied to the right arm in the supine position.

The Brachial artery was imaged in right cubital fossa and its diameter measured. (From intima media with electronic calipers) at end - systole. Systolic and diastolic velocity time integrals were measured. The arm was then occluded by raising pressure in BP cuff to atleast $50 \mathrm{~mm}$ above the systolic pressure. The Systolic and Diastolic velocity time integrals were measured within 15 seconds of release of pressure in the cuff. The brachial artery diameter was measured again at 1 minute to assess the flow mediated dilation. The ultrasound of brachial artery was continuously recorded before, during and up to 2 minutes after release of occlusion.

\section{The flow in the brachial artery calculated as:}

Base line flow $=\mathrm{Pi} * \mathrm{~d} 1 / 4 * \mathrm{HR} 1 *(\mathrm{VTIS} 1+\mathrm{VTID} 1)$

Reactive hyperemia flow $=\mathrm{Pi} * \mathrm{~d} 2 * 2 / 4 * \mathrm{HR} 2 *(\mathrm{VTIS} 2+\mathrm{VTID} 2)$ \{measured immediately after release of cuff $\}$ $\mathrm{d} 1, \mathrm{~d} 2=$ diameter of Brachial artery HR1, HR2 = heart rate VTIS1, VTIS2 = VTI Systolic VTID1, VTID2 = VTI diastolic Percentage increase in brachial artery flow was calculated as :

$\%$ Reactive hyperemia $=($ Reactive hyperemia flow - baseline flow $) * 100 /$ baseline flow Flow mediated dilation :

FMD $=(\mathrm{d} 3-\mathrm{d} 1)^{*} 100 / \mathrm{d} 1$; where $\mathrm{d} 3$ is brachial artery diameter at 1 minute of cuff release. Clinical relevance of endothelial dysfunction :

Atherosclerosis remains undetected for many years, due to lack of clinical symptoms. Once a clinical manifestation sets in, of atherosclerosis, the intervention remains essentially restricted to an improvement of symptoms and retardation of disease process. 
It is therefore of particular importance to intervene at the stage of endothelial dysfunction before the development of atherosclerosis.

\section{Methodology:-}

50 patients with impaired glucose levels Inclusion criteria:

1. Age 15-65 years

2. Subjects with impaired fasting glucose (100-125mg/dl) Exclusion criteria :

1. Below 15 years and above 65 years:

2. Diagnosed DM1 and DM2

3. Subjects with vascular diseases

4. Febrile illnesses

5. Subjects on drugs which alter flow mediated dilation of endothelium. Investigations done :

Haemoglobin , total count, differential count, ESR , FPG, Lipid profile, ECG, Color Doppler Ultrasonography.

\section{Inference:}

1. Endothelial dysfunction is seen 2 males and 1 female

2. Smoking is significantly associated with endothelial dysfunction

3. Obese people have increased risk

4. Males with waist / hip ratio greater than 1 have higher risk of premature atherosclerosis

5. High LDL and low HDL strongly correlated to impaired flow mediated vasodilatation and thus endothelial dysfunction.

\section{Results:-}

In this study endothelial dysfunction is observed in 3 patients ( $6 \%$ ) out of 50 pre diabetics, out of them 2 were males $(4.65 \%), 1$ was a female (14.85\%). People with greater BMI and HBA1C greater than $6 \%$ and males with waist / hip ratio greater than 1 were having greater chance of endothelial dysfunctoin.

\section{Conclusion:-}

In this study out of 50 pre diabetics, endothelial dysfunction was seen in $6 \%$ subjects, found more commonly in pre diabetic females $(14.85 \%)$ than in males $(4.65 \%)$. Risk factors associated with endothelial dysfunction were :

1. Increased BMI

2. Waist to hip ratio greater than 1

3. High LDL and low HDL

4. HBA1C greater than $6 \%$

5. Pre diabetics who smoke

6. Obesity in pre diabetics 